\title{
The Polytope of Non-Crossing Graphs on a Planar Point Set*
}

\author{
David Orden ${ }^{1}$ and Francisco Santos ${ }^{2}$ \\ ${ }^{1}$ Departamento de Matemáticas, \\ Universidad de Alcalá de Henares, \\ 28871 Alcalá de Henares, Spain \\ david.orden@uah.es \\ ${ }^{2}$ Departamento de Matemáticas, Estadística y Computación, \\ Universidad de Cantabria, \\ 39005 Santander, Spain \\ santosf@unican.es
}

\begin{abstract}
For any set $\mathcal{A}$ of $n$ points in $\mathbb{R}^{2}$, we define a $(3 n-3)$-dimensional simple polyhedron whose face poset is isomorphic to the poset of "non-crossing marked graphs" with vertex set $\mathcal{A}$, where a marked graph is defined as a geometric graph together with a subset of its vertices. The poset of non-crossing graphs on $\mathcal{A}$ appears as the complement of the star of a face in that polyhedron. The polyhedron has a unique maximal bounded face, of dimension $2 n_{i}+n-3$ where $n_{i}$ is the number of points of $\mathcal{A}$ in the interior of $\operatorname{conv}(\mathcal{A})$. The vertices of this polytope are all the pseudo-triangulations of $\mathcal{A}$, and the edges are flips of two types: the traditional diagonal flips (in pseudo-triangulations) and the removal or insertion of a single edge.

As a by-product of our construction we prove that all pseudo-triangulations are infinitesimally rigid graphs.
\end{abstract}

\section{Introduction and Statement of Results}

The Polyhedron of Non-Crossing Graphs. The set of (straight-line or geometric) noncrossing graphs with a given vertex set $\mathcal{A}$ in the plane is of interest in Computational Geometry, Geometric Combinatorics and related areas. In particular, much effort has been directed towards enumeration, counting and optimization on the set of maximal such graphs, that is to say, triangulations of $\mathcal{A}$.

\footnotetext{
* This research was partially supported by project BMF2001-1153 of the Spanish Dirección General de Investigación Científica.
} 
The poset structure of the family of non-crossing graphs, however, is only well understood if the points are in convex position: The non-crossing graphs containing all the hull edges are the same as the polygonal subdivisions of the convex $n$-gon and, as is well known, they form the face poset of the $(n-3)$-associahedron [14], [22].

In this paper we generalize this situation and construct from $\mathcal{A}$ a polytope whose face poset contains the poset of non-crossing graphs on $\mathcal{A}$ embedded in a very nice way. In the following statement we call interior points of $\mathcal{A}$ the points of $\mathcal{A}$ in the interior of $\operatorname{conv}(\mathcal{A})$ and we distinguish between vertices of $\operatorname{conv}(\mathcal{A})$ (extremal points) and other points of $\mathcal{A}$ in the boundary of $\operatorname{conv}(\mathcal{A})$ (semi-interior points).

Theorem 1.1. Let $\mathcal{A}$ be a set of $n$ points in the plane, not all on a line. Let $n_{i}, n_{s}$ and $n_{v}$ be the numbers of interior, semi-interior and extremal points of $\mathcal{A}$. There is a simple polytope $Y(\mathcal{A})$ of dimension $2 n_{i}+n-3$, and a face $F$ of $Y(\mathcal{A})$ (of dimension $\left.2 n_{i}+n_{v}-3\right)$ such that the complement of the star of $F$ in the face-poset of $Y(\mathcal{A})$ equals the poset of non-crossing graphs on $\mathcal{A}$ that use all the convex hull edges.

This statement deserves some words of explanation:

- Our equality of posets reverses inclusions. Maximal non-crossing graphs (triangulations of $\mathcal{A}$ ) correspond to minimal faces (vertices of $Y(\mathcal{A})$ ).

- We remind the reader that the star of a face $F$ in a polytope is the subposet of facets that contain $F$, and all their faces. In the complement of the star of $F$ we must include the polytope $Y(\mathcal{A})$ itself as an element, which corresponds to the graph with only the boundary edges of $\operatorname{conv}(\mathcal{A})$.

- If $\mathcal{A}$ is in convex position, then $Y(\mathcal{A})$ is the associahedron [14], [22]. The face $F$ is the whole polytope, whose star we must interpret as being empty.

- Our results are valid for point sets in non-general position. Our definition of noncrossing in this case is that if $q$ is between $p$ and $r$, then the edge $p r$ cannot appear in a non-crossing graph $G$, regardless of whether $q$ is incident to an edge in $G$. This definition has the slight drawback that a graph which is non-crossing in a point set $\mathcal{A}$ may become crossing in $\mathcal{A} \cup\{p\}$, but it makes maximal crossing-free graphs coincide with the triangulations of $\mathcal{A}$ (with the convention, standard in Computational Geometry, that triangulations of $\mathcal{A}$ are required to use all the points of $\mathcal{A}$ as vertices). To be consistent with this, a convex hull edge is an edge between two consecutive points of $\mathcal{A}$ in the boundary of $\operatorname{conv}(\mathcal{A})$. Since convex hull edges are irrelevant to crossingness, the poset of all non-crossing graphs on $\mathcal{A}$ is the direct product of the poset in Theorem 1.1 and a Boolean poset of rank $n_{v}+n_{s}$.

Our method provides a fully explicit facet description of $Y(\mathcal{A})$. It lives in $\mathbb{R}^{3 n}$ and is defined by the three linear equalities (1) and the $\left(\begin{array}{l}n \\ 2\end{array}\right)+n$ linear inequalities (4) and (5) of Section 3, with some of them turned into equalities. (With one exception: for technical reasons, if $\mathcal{A}$ contains three collinear boundary points we need to add extra points to its exterior and obtain $Y(\mathcal{A})$ as a face of the polytope $Y\left(\mathcal{A}^{\prime}\right)$ of the extended point set

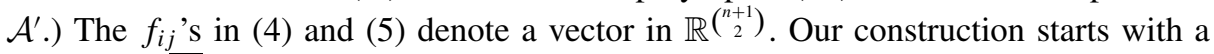
linear cone $\overline{Y_{0}}(\mathcal{A})$ (Definition 3.1) whose facets are then translated using the entries of $f$ to produce a polyhedron $\overline{Y_{f}}(\mathcal{A})$, of which the polytope $Y(\mathcal{A})=Y_{f}(\mathcal{A})$ is the unique maximal bounded face. The proof of Theorem 1.1 goes by analyzing the necessary 
and sufficient conditions for $f$ to produce a polytope with the desired properties and then proving the existence of valid choices of $f$. In particular, Theorem 3.8 shows one specific valid choice. This is essentially the same approach used in [19] for the polytope of pointed non-crossing graphs constructed there. We come back to the relation between [19] and the present paper later in this Introduction.

Triangulations as a Polytope. Mapping the instances of a combinatorial object to the vertices of a certain polytope is useful for optimization and enumeration purposes. In the case of triangulations of a (not necessarily two-dimensional) point set, two such polytopes have been used in the past; the "secondary polytope" [5] and the "universal polytope" [8] of the point set. Our construction adds to these two, but it has one advantage: We have an explicit facet description of the polytope. In the secondary polytope, facets correspond to the coarse polygonal subdivisions of $\mathcal{A}$, which have no easy characterization. In the universal polytope, the facet description in [8] gives only a linear programming relaxation of the polytope, which makes integer programming necessary in order to optimize linear functionals on it. It has to be noted, also, that the secondary polytope does not contain all the triangulations of a point set as vertices. Only the so-called regular or generalized Delaunay triangulations.

That the poset we are interested in is not the whole poset of faces of the polytope $Y(\mathcal{A})$ may seem a serious drawback for using it as a tool for enumeration of all the triangulations of a planar point set. However, the subposet we are interested in is not just a subposet. Being the complement of the star of a face $F$ has theoretical and practical implications. On the one hand, it implies that the poset is a shellable ball of dimension $2 n_{i}+n-4$, since there is a shelling order ending in the facets that contain $F$. On the other hand, the part of the boundary of $Y(\mathcal{A})$ that we are interested in becomes the (strict) lower envelope of a convex polyhedron via any projective transformation that sends a supporting hyperplane of $F$ to infinity.

The Polytope of Pseudo-Triangulations. Actually, the set of all the vertices of the polytope $Y(\mathcal{A})$ is interesting in its own merits:

Theorem 1.2. The vertex set of the polytope $Y(\mathcal{A})$ of Theorem 1.1 is in bijection to the set of all pseudo-triangulations of $\mathcal{A}$. The 1-skeleton of $Y(\mathcal{A})$ is the graph of flips between them.

A pseudo-triangulation of $\mathcal{A}$ is a subdivision of $\operatorname{conv}(\mathcal{A})$ into pseudo-triangles using $\mathcal{A}$ as the vertex set, and a pseudo-triangle is a perhaps non-convex polygon with three corners (non-reflex vertices). As an example, Fig. 1 shows the pseudo-triangulations of a set of four extremal points and one interior point, with the graph of flips between them embedded as (a Schlëgel diagram of) a four-dimensional polytope. The face $F$ of Theorem 1.1 is the three-dimensional facet on which the Schlëgel diagram has been performed. Why the interior point is "unmarked" in the three triangulations of the point set and "marked" in the other eight pseudo-triangulations is explained in Section 2.

Pseudo-triangulations, first introduced by Pocchiola and Vegter around 1995 (see [17]), have by now been used in many Computational Geometry applications, among them visibility [16]-[18], [20], ray shooting [10] and kinetic data structures [1], [13]. 


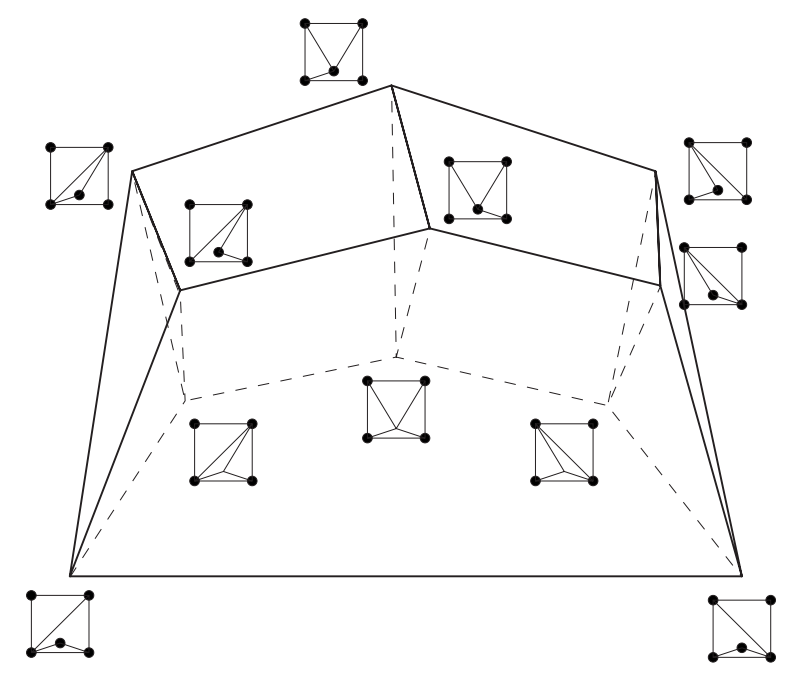

Fig. 1. The pseudo-triangulations of a point set form a polytope.

Streinu [21] introduced the minimum or pointed pseudo-triangulations (p.p.t.'s for short), discovered certain surprising relations of them to structural rigidity of non-crossing graphs and used them to prove Carpenter's Rule Theorem (the first proof of which was given shortly before by Connelly et al. [6], [7]). P.p.t.'s can also be described as the maximal non-crossing and pointed graphs. See all the relevant definitions in Section 2.

Relation to the Previous Paper [19]. Rote et al. in [19], using these rigid theoretic properties of p.p.t.'s, constructed a polytope $X(\mathcal{A})$ whose vertex set is precisely the set of p.p.t.'s of a given point set. The present paper can be considered a sequel to that paper. The steps in our construction are essentially the same as there and the polytope $X(\mathcal{A})$ of [19] equals the face $F$ of $Y(\mathcal{A})$ that appears in the statement of Theorem 1.1. The two novelties in our results are that we extend that construction to cover all pseudotriangulations and all non-crossing graphs instead of only pointed ones, and also that we show how the method can be adapted to point sets in non-general position, with a suitable definition of pseudo-triangulation for such point sets (Definition 5.1).

Technically speaking, the three new ingredients here, not present in [19], are:

- We use a generalized definition of flip between pseudo-triangulations that allows us to increase or decrease the number of edges (see Definition 2.5 for point sets in general position and Definition 5.5 and Figs. 9 and 10 for point sets with collinearities). In the case of general position, the same definition has been independently considered in [2], where flips between pseudo-triangulations are related to locally convex functions and geometric flips between polyhedral terrains.

For p.p.t.'s, our flips restrict to the traditional "edge-flips" introduced in [21] and used in [19]. Our flips are also related to the flips between "pseudo-triangulations of convex bodies" [17] as follows: Pocchiola (personal communication) has shown 
that taking a sufficiently small convex body around each point of a point set $\mathcal{A}$, our graph of flips is obtained from the one in [17] by contraction of certain edges.

- Suggested by the properties of the graph of flips (it is regular of degree equal to the number of "edges plus pointed vertices" of any given pseudo-triangulation of $\mathcal{A}$ ), we introduce a formalism in which the "pointedness" of any particular vertex of $\mathcal{A}$ in a graph $G$ plays the same role as the presence of an edge. This led to the concept of a "marked non-crossing graph" which is a non-crossing graph with some of its pointed vertices "marked".

- In order to extend the results of [19] we needed a way to make "marks" enter into the rigidity theoretic picture used there: to be precise, we needed to work with "infinitesimal motions" in which each vertex has a three-dimensional "velocity vector" attached to it. More importantly, we needed to define expansiveness and equilibrium for these "motions", in such a way as to produce exactly the signs given in Lemma 4.3 for the unique equilibrium stress on the complete marked graph on four points. It took us some time to find the right notion of expansiveness. As sometimes happens, the final solution (given in (2) and (3)) turned out to be simpler than other approaches we tried before.

Constrained Pseudo-Triangulations. It is sometimes convenient to study pseudo-triangulations or non-crossing graphs on $\mathcal{A}$ that contain a specified subset of edges. For example, the pseudo-triangulations of a non-convex polygon $P$ are in bijection with pseudo-triangulations of the point set $\mathcal{A}=\operatorname{vertices}(P)$ which use all the boundary edges of $P$ and any (arbitrarily chosen) triangulation of $\operatorname{conv}(P) \backslash P$. We can even allow for additional interior points of $P$ to be used as vertices (a situation called an "augmented polygon" in [2]).

We want to emphasize that our results apply to these and other cases: since each facet of $Y(\mathcal{A})$ contains the graphs that use a certain edge, the graphs that extend a certain given graph $G$ correspond to a certain face of $Y(\mathcal{A})$. We can even prescribe vertices to be pointed since, as said above, "pointedness of a vertex plays the same role as the presence of an edge".

Corollary 1.3. Let $G$ be a non-crossing graph on $\mathcal{A}$ with $k$ non-boundary edges (edges not in the boundary of $\operatorname{conv}(\mathcal{A}))$. Then the set of pseudo-triangulations of $\mathcal{A}$ that contain $G$ as a subgraph is the vertex set of a simple polytope of dimension $2 n_{i}+n-3-k$, whose 1-skeleton is the graph of flips between them. In particular, the pseudo-triangulations of a non-convex polygon $P$ with $n$ vertices are the vertex set of a polytope of dimension $n-3$.

This result was left as an open question in [2], where the case of a simple polygon with no interior vertices was proved. As an example, Fig. 2 shows the (three-dimensional) polytope of pseudo-triangulations of a certain non-convex hexagon.

Pseudo-Triangulations Are Rigid. Our construction also has rigid-theoretic consequences. A generically rigid graph (for dimension 2) is a graph which becomes rigid in almost all its straight-line embeddings in the plane. Generically rigid graphs need at least 


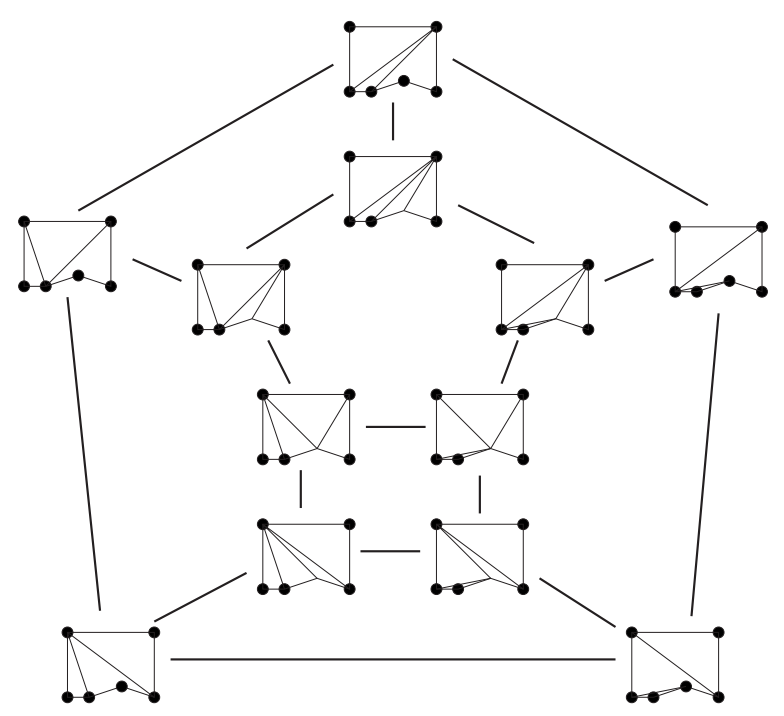

Fig. 2. The pseudo-triangulations of a (perhaps non-convex) polygon form a polytope too.

$2 n-3$ edges, because that is the number of degrees of freedom of $n$ points in the plane (after neglecting rigid motions). Generically rigid graphs with exactly $2 n-3$ edges are called isostatic and they admit the following characterization, due to Laman (see, for example, [11]): they are the graphs with $2 n-3$ edges and with the property that any subset of $k \leq n-2$ vertices is incident to at least $2 k$ edges. Using this characterization, Streinu [21] proved that every pointed pseudo-triangulation is an isostatic graph. We have the following generalization:

Theorem 1.4. Let $T$ be a pseudo-triangulation of a planar point set $\mathcal{A}$ in general position. Let $G$ be its underlying graph. Then:

(1) $G$ is infinitesimally rigid, hence rigid and generically rigid.

(2) There are at least $2 k+3 l$ edges of $T$ incident to any subset of $k$ pointed plus $l$ non-pointed vertices of $T$ (assuming $k+l \leq n-2$ ).

In this statement, "general position" can actually be weakened to "no three boundary points of $\mathcal{A}$ are collinear". In the presence of boundary collinearities, non-rigid pseudotriangulations (for our definition) exist. For example, only six of the fourteen pseudotriangulations of the point set of Fig. 10 are rigid.

If we recall that a pseudo-triangulation with $k$ non-pointed vertices has exactly $2 n-$ $3+k$ edges (see Proposition 2.2), then Theorem 1.4 has the consequence that the space of equilibrium stresses on a pseudo-triangulation has exactly dimension $k$. This fact also follows from the results of [2].

The property in part (2) of Theorem 1.4 also has some interest. It is a natural generalization of the Laman property of pointed pseudo-triangulations, and it has been used in [15] to prove the following statement. The "isostatic" case of it was proved in [12]: 
Theorem 1.5 [15]. A planar graph is generically rigid if and only if it can be embedded as a pseudo-triangulation.

Two Open Questions.

- It is proved in [3] that the graph of flips between pseudo-triangulations of a point set $\mathcal{A}$ (that is, the 1-skeleton of our polytope) has diameter bounded by $O(n \log (n))$. The same bound for the graph of pointed pseudo-triangulations (the 1-skeleton of the face $F$ ) has been obtained in [4]. Is there a general upper bound of the form $O(d \log d)$ for the diameter of the graph of any face of $Y(\mathcal{A})$, where $d$ denotes the dimension of that face? Even more, is there an $O(d)$ upper bound? (This is open even for the whole polytope, or for the polytope of pointed pseudo-triangulations.)

Essentially, we are asking for an upper bound on the diameter of the graph of flips between pseudo-triangulations constrained by any given graph $G$ on $\mathcal{A}$, and expect the bound to depend only on the number of edges needed to complete $G$ to a pseudo-triangulation.

- Are non-crossing graphs on $\mathcal{A}$ the face poset of a polyhedron? A naive answer would be that such a polyhedron is obtained by just deleting from the facet definition of $Y(\mathcal{A})$ those facets containing $F$. However, this does not work even in the point set with a single point in general position in the interior of a quadrilateral (the one in Fig. 1). Using the equations that define the polytope it can be checked that the removal of the facet $F$ in this example gives a polyhedron with two extra vertices, not present in $Y_{f}(\mathcal{A})$.

\section{The Graph of All Pseudo-Triangulations of $\mathcal{A}$}

Throughout this section, $\mathcal{A}$ denotes a set of $n$ points in general position in the plane, $n_{i}$ of them in the interior of $\operatorname{conv}(\mathcal{A})$ and $n_{v}$ in the boundary. Point sets in non-general position are studied in Section 5.

Definition 2.1. A pseudo-triangle is a simple polygon with only three convex vertices (called corners) joined by three inward convex polygonal chains (called pseudo-edges of the pseudo-triangle).

A pseudo-triangulation of $\mathcal{A}$ is a geometric non-crossing graph with vertex set $\mathcal{A}$ and which partitions $\operatorname{conv}(\mathcal{A})$ into pseudo-triangles.

Part (a) of Fig. 3 shows a pseudo-triangle. Parts (b) and (c) show two pseudotriangulations.

Since the maximal non-crossing graphs on $\mathcal{A}$ (the triangulations of $\mathcal{A}$ ) are a particular case of pseudo-triangulations, they are the maximal pseudo-triangulations. As is well known, they all have $2 n_{v}+3 n_{i}-3$ edges. It turns out that the pseudo-triangulations with the minimum possible number of edges are also very interesting from different points of view. We recall that a vertex of a geometric graph is called pointed if all its incident edges span an angle smaller than $\pi$ from that vertex. The graph itself is called pointed if all its vertices are pointed. The following statement comes originally from [21] and a proof can also be found in [19]. 


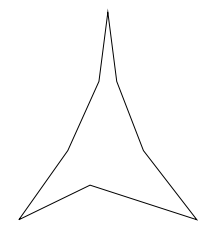

(a)

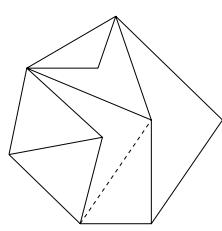

(b)

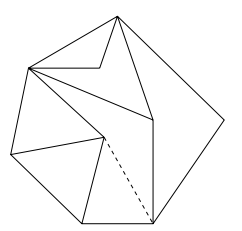

(c)

Fig. 3. (a) A pseudo-triangle. (b) A pointed pseudo-triangulation. (c) The dashed edge in (b) is flipped, giving another pointed pseudo-triangulation.

Proposition 2.2 [21]. Let $\mathcal{A}$ be a planar point set as above. Then:

1. Every pseudo-triangulation of $\mathcal{A}$ with $n_{\gamma}$ non-pointed vertices and $n_{\varepsilon}$ pointed vertices has $2 n-3+n_{\gamma}=3 n-3-n_{\varepsilon}$ edges.

2. Every pointed and planar graph on $\mathcal{A}$ has at most $2 n-3$ edges, and is contained in some pointed pseudo-triangulation of $\mathcal{A}$.

Part 1 implies that, among pseudo-triangulations of $\mathcal{A}$, pointed ones have the minimum possible number of edges. For this reason they are sometimes called minimum pseudotriangulations. Part 2 says that pointed pseudo-triangulations coincide with maximal non-crossing and pointed graphs.

Another crucial property of pseudo-triangulations is the existence of a natural notion of flip. Let $e$ be an interior edge in a pseudo-triangulation $T$ of $\mathcal{A}$ and let $\sigma$ be the union of the two pseudo-triangles incident to $e$. We regard $\sigma$ as a graph, one of whose edges is $e$. We can consider $\sigma \backslash e$ to be a (perhaps degenerate) polygon, with a well-defined boundary cycle; in degenerate cases some edges and vertices may appear twice in the cycle. See an example of what we mean in Fig. 4, in which the cycle of vertices is pqrstsu and the cycle of edges is $p q, q r, r s, s t, t s, s u, u p$. As in any polygon, each (appearance of a) vertex in the boundary cycle of $\sigma \backslash e$ is either concave or convex. In the figure there are four convex vertices (corners), namely $r$, the second appearance of $s, u$ and $q$. Then:

\section{Lemma 2.3.}

1. $\sigma \backslash e$ has either three or four corners.

2. It has three corners if and only if exactly one of the two endpoints of $e$ is pointed in $\sigma$. In this case $T \backslash e$ is still a pseudo-triangulation.
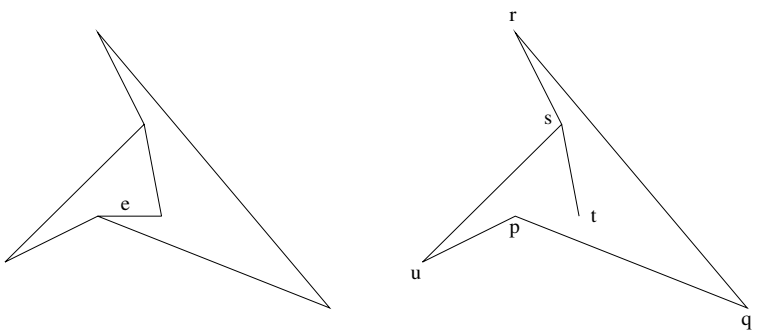

Fig. 4. The region $\sigma \backslash e$ is a degenerate polygon with four corners. 
3. It has four corners if and only if both endpoints of e are pointed in $\sigma$. In this case $T \backslash e$ is not a pseudo-triangulation and there is a unique way to insert an edge in $T \backslash e$ to obtain another pseudo-triangulation.

Proof. Let $v_{1}$ and $v_{2}$ be the two endpoints of $e$. For each $v_{i}$, one of the following three things occur: (a) $v_{i}$ is not-pointed in $\sigma$, in which case it is a corner of the two pseudotriangles incident to $e$ and is not a corner of $\sigma \backslash e$; (b) $v_{i}$ is pointed in $\sigma$ with the big angle exterior to $\sigma$, in which case it is a corner of both pseudo-triangles and of $\sigma \backslash e$ as well, or (c) $v_{i}$ is pointed with its big angle interior, in which case it is a corner in only one of the two pseudo-triangles and not a corner in $\sigma \backslash e$.

In case (a), $v_{i}$ contributes two more corners to the two pseudo-triangles than to $\sigma \backslash e$. In the other two cases, it contributes one more corner to the pseudo-triangles than to $\sigma \backslash e$. Since the two pseudo-triangles have six corners in total, $\sigma \backslash e$ has four, three or two corners depending on whether both, one or none of $v_{1}$ and $v_{2}$ are pointed in $\sigma$. The case of two corners is clearly impossible, which finishes the proof of part 1 . Part 2 only says that "degenerate pseudo-triangles" cannot appear.

Part 3 is equivalent to saying that a pseudo-quadrangle (even a degenerate one) can be divided into two pseudo-triangles in exactly two ways. Indeed, these two partitions are obtained drawing the geodesic arcs between two opposite corners. Such a geodesic path consists of a unique interior edge and (perhaps) some boundary edges.

Parts 2 and 3 of the above lemma will define two different types of flips in a pseudotriangulation. The inverse of the first one is the insertion of an edge, in case this produces a pseudo-triangulation. It is easy to describe exactly when this happens:

Lemma 2.4. Let $T$ be a pseudo-triangle with $k$ non-corners. Then every interior edge dividing $T$ into two pseudo-triangles makes non-pointed exactly one non-corner. Moreover, there are exactly $k$ such interior edges, each making non-pointed a different noncorner.

Proof. The first sentence follows from Lemma 2.3, which says that exactly one of the two endpoints of the edge inserted is pointed (after the insertion). For each non-corner, pointedness at the other end of the edge implies that the edge is the one that arises in the geodesic arc that joins that non-corner to the opposite corner. This proves uniqueness and existence.

Definition 2.5 (Flips in Pseudo-Triangulations). Let $T$ be a pseudo-triangulation. We call flips in $T$ the following three types of operations, all producing pseudo-triangulations. See examples in Fig. 5:

- (Deletion flip.) The removal of an edge $e \in T$, if $T \backslash e$ is a pseudo-triangulation.

- (Insertion flip.) The insertion of an edge $e \notin T$, if $T \cup e$ is a pseudo-triangulation.

- (Diagonalflip.) The exchange of an edge $e \in T$, if $T \backslash e$ is not a pseudo-triangulation, for the unique edge $e^{\prime}$ such that $(T \backslash e) \cup e^{\prime}$ is a pseudo-triangulation.

The graph of pseudo-triangulations of $\mathcal{A}$ has as vertices all the pseudo-triangulations of $\mathcal{A}$ and as edges all flips of any of the types. 

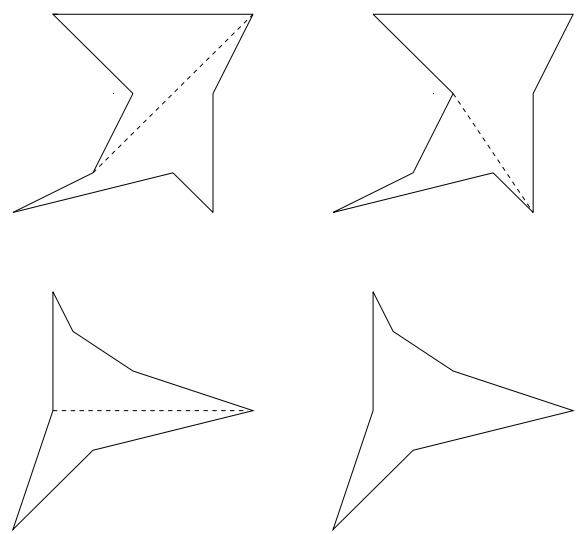

Fig. 5. (Top) A diagonal flip. (Bottom) An insertion-deletion flip.

A proof of the following can also be found in [2]:

Proposition 2.6. The graph of pseudo-triangulations of $\mathcal{A}$ is connected and regular of degree $3 n_{i}+n_{v}-3=3 n-2 n_{v}-3$.

Proof. There is one diagonal or deletion flip for each interior edge, giving a total of $3 n-3-n_{\varepsilon}-n_{v}$ by Proposition 2.2. There are as many insertion flips as pointed interior vertices by Lemma 2.4 , giving $n_{\varepsilon}-n_{v}$.

To establish connectivity, let $p$ be a point on the convex hull of $\mathcal{A}$. By induction, we can assume that the pseudo-triangulations of $\mathcal{A}$ with no interior edge at $p$ are connected by flips (they coincide with the pseudo-triangulations of $\mathcal{A} \backslash\{p\}$ together with the two tangents from $p$ to $\mathcal{A} \backslash\{p\}$ ). In a pseudo-triangulation with interior edges at $p$, interior edges incident to $e$ can be flipped out one by one.

Remark 2.7. It is an immediate consequence of Lemma 2.3 that every interior edge in a pointed pseudo-triangulation is flippable. This shows that the graph of diagonal flips between pointed pseudo-triangulations of $\mathcal{A}$ is regular of degree $2 n_{i}+n_{v}-3$, a crucial fact in [19]. The diameters of the graphs of all pseudo-triangulations and of pointed pseudo-triangulations are studied in [3] and [4], respectively. They are both in $O(n \log n)$.

As another remark, observe that if a pseudo-triangulation $T^{\prime}$ is obtained from another one $T$ by a deletion flip followed by an insertion flip, then $T$ and $T^{\prime}$ differ by a single edge and we do not consider them joined by a flip.

Marked Non-Crossing Graphs on $\mathcal{A}$. As happened with pointed pseudo-triangulations, Proposition 2.6 suggests that the graph of pseudo-triangulations of $\mathcal{A}$ may be the skeleton of a simple polytope of dimension $3 n_{i}+n_{v}-3$. As a step towards this result we first look at what the face poset of such a polytope should be. The polytope being simple means that we want to regard each pseudo-triangulation $T$ as the upper bound element in a Boolean poset of order $3 n-3-2 n_{v}$. This number equals, by Proposition 2.2, the 
number of interior edges plus interior pointed vertices in $T$. This suggests the following definitions:

Definition 2.8. A marked graph on $\mathcal{A}$ is a geometric graph with vertex set $\mathcal{A}$ together with a subset of its vertices, that we call "marked". We call a marked graph non-crossing if it is non-crossing as a graph and marks arise only in pointed vertices. We call a noncrossing marked graph fully marked if it is marked at all pointed vertices. If, in addition, it is a pseudo-triangulation, then we call it a fully marked pseudo-triangulation, abbreviated as f.m.p.t.

Marked graphs form a poset by inclusion of both the sets of edges and of marked vertices. We say that a marked graph contains the boundary of $\mathcal{A}$ if it contains all the convex hull edges and convex hull marks.

Observe that there is a natural bijection between pseudo-triangulations (graphs) and f.m.p.t.'s (which turn out to be the maximal non-crossing marked graphs). In other words, the face poset of our polytope should be the poset of non-crossing marked graphs on $\mathcal{A}$ that contain the boundary. Indeed, this poset has the right " 1 -skeleton" and the right lower ideal below every f.m.p.t. (a Boolean lattice of order $3 n-3-2 n_{v}$ ).

The graph of pseudo-triangulations of $\mathcal{A}$ of Definition 2.5 has a natural interpretation as a graph of "flips between fully marked pseudo-triangulations": a diagonal flip exchanges one edge to another one, keeping pointedness (and hence marks) of every vertex. The edge-deletion and edge-insertion flips exchange an edge for a mark, and vice versa (Fig. 6). Figures 1 and 2 were drawn with this interpretation in mind.

The following results are mere rephrasings of the corresponding statements for noncrossing graphs and pseudo-triangulations, and are stated here for future reference:

Proposition 2.9. With the previous definitions:

1. Every marked pseudo-triangulation of $\mathcal{A}$ with $n_{\gamma}$ non-pointed vertices, $n_{\varepsilon}$ pointed vertices and $n_{m}$ marked vertices, has $2 n-3+n_{\gamma}+n_{m}=3 n-3-n_{\varepsilon}+n_{m}$ edges plus marks. In particular, all f.m.p.t.'s have $3 n-3$ edges plus marks, $3 n-3-2 n_{v}$ of them interior.

2. (Flips in marked pseudo-triangulations) In an f.m.p.t. $T$ of $\mathcal{A}$, every interior edge or interior mark can be flipped; that is to say, once an interior edge or mark is removed from $T$ there is a unique edge or mark (different from the removed one) that can be added in order to get another f.m.p.t. of $\mathcal{A}$. In particular, the graph of flips between f.m.p.t.'s of $\mathcal{A}$ is connected and regular of degree $3 n-3-2 n_{v}$.
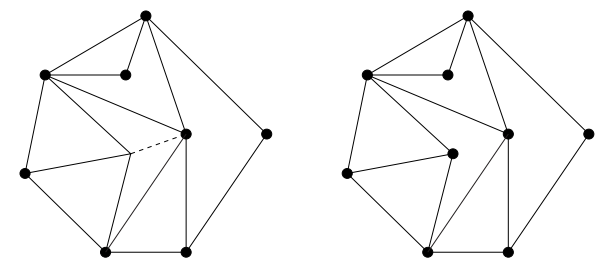

Fig. 6. Two marked pseudo-triangulations (with marks represented by dots) related by a flip. An edge from the left is switched to a mark on the right. 


\section{The Polyhedron of Marked Non-Crossing Graphs on $\mathcal{A}$}

Here we construct the polytope and polyhedron referred to in the Introduction. In the first part of this section we do not assume $\mathcal{A}$ to be in general position. Only after Definition 3.7 do we need general position, among other reasons because we have not yet defined marked non-crossing graphs or pseudo-triangulations for point sets in special position. That is done in Section 5.

The setting for our construction is close to the rigid-theoretic one used in [19]. There the polytope to be constructed is embedded in the space $\mathbb{R}^{2 n-3}$ of all infinitesimal motions of the $n$ points $p_{1}, \ldots, p_{n}$. The space has dimension $2 n-3$ because the infinitesimal motion of each point produces two coordinates (an infinitesimal velocity $v_{i} \in \mathbb{R}^{2}$ ) but global translations and rotations produce a three-dimensional subspace of trivial motions which are neglected. Formally, this can be done by a quotient $\mathbb{R}^{2 n} / M_{0}$, where $M_{0}$ is the three-dimensional subspace of trivial motions, or it can be done by fixing three of the $2 n$ coordinates to be zero. For example, if the points $p_{1}$ and $p_{2}$ do not lie in the same horizontal line, one can take

$$
v_{1}^{1}=v_{1}^{2}=v_{2}^{1}=0 .
$$

In our approach, we consider a third coordinate $t_{i}$ for each point, related to the "marks" discussed in the previous paragraphs, or to pointedness of the vertices.

That is to say, given a set of $n$ points $\mathcal{A}=\left\{p_{1}, \ldots, p_{n}\right\}$ in $\mathbb{R}^{2}$, we consider the following $(3 n-3)$-dimensional space of "infinitesimal motions":

$$
S:=\left\{\left(v_{1}, \ldots, v_{n}, t_{1}, \ldots, t_{n}\right) \in\left(\mathbb{R}^{2}\right)^{n} \times \mathbb{R}^{n}: v_{1}^{1}=v_{1}^{2}=v_{2}^{1}=0\right\} \subset \mathbb{R}^{3 n} .
$$

In it we consider the following $\left(\begin{array}{l}n \\ 2\end{array}\right)+n$ linear inequalities:

$$
H_{i j}^{+}:=\left\{(v, t) \in S:\left\langle p_{i}-p_{j}, v_{i}-v_{j}\right\rangle-\left|p_{i}-p_{j}\right|\left(t_{i}+t_{j}\right) \geq 0\right\}
$$

and

$$
H_{0 j}^{+}:=\left\{(v, t) \in S: t_{j} \geq 0\right\} .
$$

We denote their boundary hyperplanes by $H_{i, j}$ and $H_{0, j}$.

Definition 3.1. We call the positive region of the above hyperplane arrangement the expansion cone of $\mathcal{A}$ and denote it $\overline{Y_{0}}(\mathcal{A})$ :

$$
\overline{Y_{0}}(\mathcal{A}):=\bigcap_{i, j \in\{0,1, \ldots, n\}} H_{i j}^{+} .
$$

When clear from the context we omit the point set $\mathcal{A}$ and use just $\overline{Y_{0}}$.

Remark 3.2. Observe that the equations defining $\overline{Y_{0}}$ imply that for every $i, j$,

$$
\left\langle p_{i}-p_{j}, v_{i}-v_{j}\right\rangle \geq\left|p_{i}-p_{j}\right|\left(t_{i}+t_{j}\right) \geq 0 .
$$

In particular, in order for $\left(v_{1}, \ldots, v_{n}, t_{1}, \ldots, t_{n}\right)$ to be expansive in our sense, the vector 
$\left(v_{1}, \ldots, v_{n}\right)$ must be an expansive infinitesimal motion of the point set, in the standard sense.

Lemma 3.3. The polyhedron $\overline{Y_{0}}(\mathcal{A})$ has full dimension $3 n-3$ in $S \subset \mathbb{R}^{3 n}$ and it is a pointed polyhedral linear cone. (Here, "pointed" means "having a vertex" or, equivalently, "containing no whole line”.)

Proof. The vector $(v, t)$ with $v_{i}:=p_{i}, t_{i}:=\min _{k, l}\left\{\left|p_{k}-p_{l}\right|\right\} / 4$ satisfies all the inequalities (2) and (3) strictly. In order to obtain a point in $S$ we add to it a suitable infinitesimal trivial motion.

To prove that the cone is pointed, suppose that it contains two opposite vectors $(v, t)$ and $-(v, t)$. Equivalently, that $(v, t)$ lies in all the hyperplanes $H_{i, j}$ and $H_{0, i}$. That is to say, $t_{i}=0$ for every $i$ and

$$
\left\langle v_{j}-v_{i}, p_{j}-p_{i}\right\rangle=0
$$

for all $i, j$. These last equations say that $\left(v_{1}, \ldots, v_{n}\right)$ is an infinitesimal flex of the complete graph on $\mathcal{A}$. Since the complete graph on every full-dimensional point set is infinitesimally rigid, $\left(v_{1}, \ldots, v_{n}\right)$ is a trivial motion and (1) implies that the motion is zero.

An edge $p_{i} p_{j}$ or a point $p_{i}$ are called tight for a certain vector $(v, t) \in \overline{Y_{0}}$ if $(v, t)$ lies in the corresponding hyperplane $H_{i, j}$ or $H_{0, i}$. For any $(v, t)$, we denote by $T(v, t)$ the marked graph of tight edges for $(v, t)$ with marks at tight points for $(v, t)$. We call it the supporting graph of $(v, t)$.

Lemma 3.4. Let $(v, t) \in \overline{Y_{0}}$. If $T(v, t)$ contains the boundary edges and vertices of a convex polygon, then $v_{l}=0$ and $t_{l}=0$ for every point $p_{l}$ in the interior of the polygon. Therefore, $T(v, t)$ contains the complete marked graph on the set of vertices and interior points of the polygon.

Observe that this statement says nothing about points in the relative interior of a boundary edge, if the polygon has collinear points in its boundary. Indeed, such points may have a non-zero $v_{l}$, namely, the exterior normal to the boundary edge containing the point.

Proof. The hypotheses are equivalent to $t_{i}=0$ and $\left\langle p_{i}-p_{j}, v_{i}-v_{j}\right\rangle=0$ for all the boundary vertices $p_{i}$ and boundary edges $p_{i} p_{j}$ of the convex polygon.

Then the infinitesimal expansive motion $v=\left(v_{1}, \ldots, v_{n}\right)$ also preserves distances between non-consecutive polygon vertices, because otherwise it would decrease the interior angle at some polygon vertex $p_{i}$ and it would make its adjacent boundary vertices get closer, in contradiction with (2). Hence, $v$ is a translation or rotation of the polygon boundary which, by (1), is zero.

In these conditions, if $v_{k} \neq 0$ for some $p_{k}$ interior to the polygon, then $p_{k}$ gets closer to some boundary vertex, that using $t_{k} \geq 0$ contradicts (2) again. Therefore, $v_{l}=0$ for 
every point $p_{l}$ enclosed in the polygon. Then (2) taking $p_{j}=p_{l}$ and $p_{i}$ in the boundary of the polygon implies that $t_{l} \leq 0$. Together with (3) taking again $p_{j}=p_{l}$ this implies $t_{l}=0$.

Obviously, $\overline{Y_{0}}$ is not the polyhedron we are looking for, since its face poset does not have the desired combinatorial structure; it has a unique vertex while $\mathcal{A}$ may have more than only one f.m.p.t. The right polyhedron for our purposes is going to be a convenient perturbation of $\overline{Y_{0}}$ obtained by translation of its facets.

Definition 3.5. For each $f \in \mathbb{R}_{\left(\begin{array}{c}n+1 \\ 2\end{array}\right)}$ (with entries indexed $f_{i, j}$, for $i, j \in\{0, \ldots, n\}$ ) we denote by $\overline{Y_{f}}(\mathcal{A})$ the polyhedron defined by the $\left(\begin{array}{l}n \\ 2\end{array}\right)$ equations

$$
\left\langle p_{i}-p_{j}, v_{i}-v_{j}\right\rangle-\left|p_{i}-p_{j}\right|\left(t_{i}+t_{j}\right) \geq f_{i j}
$$

for every $p_{i}, p_{j} \in \mathcal{A}$ and the $n$ equations

$$
t_{j} \geq f_{0 j}, \quad \forall p_{i} \in \mathcal{A} .
$$

We call it the polyhedron of expansions constrained by $f$.

From Lemma 3.3, we conclude that:

Corollary 3.6. $\overline{Y_{f}}(\mathcal{A})$ is a $(3 n-3)$-dimensional unbounded polyhedron with at least one vertex, for any $f$.

In the rest of this section and in Section 4 we assume $\mathcal{A}$ to be in general position. As before, to each feasible point $(v, t) \in \overline{Y_{f}}$ we associate the marked graph consisting of edges and vertices for which (4) and (5) are tight on $(v, t)$. Similarly, to a face $F$ of $\overline{Y_{f}}$ we associate the tight marked graph of any of its relative interior points. This gives an (order-reversing) embedding of the face poset of $\overline{Y_{f}}$ into the poset of all marked graphs of $\mathcal{A}$. Our goal is to show that for certain choices of the constraint parameters $f$, the face poset of $\overline{Y_{f}}$ coincides with that of non-crossing marked graphs on $\mathcal{A}$.

Definition 3.7. We define a choice of the constants $f$ to be valid if the tight marked graph $T(F)$ of every face $F$ of $\overline{Y_{f}}$ is non-crossing.

The proof that valid choices exist for any point set is postponed to Section 4, in order not to interrupt the current flow of ideas. In particular, Corollary 4.5 implies that the following explicit choice is valid:

Theorem 3.8. The choice $f_{i j}:=\operatorname{det}\left(O, p_{i}, p_{j}\right)^{2}, f_{0 j}:=0$ is valid.

The main statement in the paper is then:

Theorem 3.9 (The Polyhedron of Marked Non-Crossing Graphs). If $f$ is a valid choice of parameters, then $\overline{Y_{f}}$ is a simple polyhedron of dimension $3 n-3$ whose face poset equals (the opposite of) the poset of non-crossing marked graphs on $\mathcal{A}$. In particular: 
(a) Vertices of the polyhedron are in 1-to-1 correspondence with f.m.p.t.'s of $\mathcal{A}$.

(b) Bounded edges correspond to flips of interior edges or marks in f.m.p.t.'s, i.e., to f.m.p.t.'s with one interior edge or mark removed.

(c) Extreme rays correspond to f.m.p.t.'s with one convex hull edge or mark removed.

Proof. By Corollary 3.6, every vertex $(v, t)$ of $\overline{Y_{f}}$ has at least $3 n-3$ incident facets. By Proposition 2.9, if $f$ is valid, then the marked graph of any vertex of $\overline{Y_{f}}$ has exactly $3 n-3$ edges plus marks and is an f.m.p.t. This also implies that the polyhedron is simple. If we prove that all the f.m.p.t.'s appear as vertices of $\overline{Y_{f}}$ we finish the proof, because then the face poset of $\overline{Y_{f}}$ will have the right minimal elements and the appropriate upper ideals of minimal elements (the Boolean lattices of subgraphs of f.m.p.t.'s) to coincide with the poset of non-crossing marked graphs on $\mathcal{A}$.

That all f.m.p.t.'s appear follows from connectedness of the graph of flips: Starting with any given vertex of $\overline{Y_{f}}$, corresponding to a certain f.m.p.t. $T$ of $\mathcal{A}$, its $3 n-3$ incident edges correspond to the removal of a single edge or mark in $T$. Moreover, if the edge or mark is not in the boundary, Lemma 3.4 implies that the edge (of $\overline{Y_{f}}$ ) corresponding to it is bounded because it collapses to the origin in $\overline{Y_{0}}$. Then this edge connects the original vertex of $\overline{Y_{f}}$ to another one which can only be the f.m.p.t. given by the flip in the corresponding edge or mark of $T$. Since this happens for all vertices, and since all f.m.p.t.'s are reachable from any other one by flips, we conclude that they all appear as vertices.

From Theorems 3.8 and 3.9 it is easy to conclude the statements in the Introduction. The following is actually a more precise statement implying both:

Theorem 3.10 (The Polytope of All Pseudo-Triangulations). Let $Y_{f}(\mathcal{A})$ be the face of $\overline{Y_{f}}(\mathcal{A})$ defined by turning into equalities equations (4) and (5) which correspond to convex hull edges or convex hull points of $\mathcal{A}$, and assume $f$ to be a valid choice. Then:

1. $Y_{f}(\mathcal{A})$ is a simple polytope of dimension $2 n_{i}+n-3$ whose 1 -skeleton is the graph of pseudo-triangulations of $\mathcal{A}$. (In particular, it is the unique maximal bounded face of $\overline{Y_{f}}(\mathcal{A})$ ).

2. Let $F$ be the face of $Y_{f}(\mathcal{A})$ defined by turning into equalities the remaining equations (5). Then the complement of the star of $F$ in the face-poset of $Y_{f}(\mathcal{A})$ equals the poset of non-crossing graphs on $\mathcal{A}$ that use all the convex hull edges.

Proof. (1) That $Y_{f}(\mathcal{A})$ is a bounded face follows from Lemma 3.4 (it collapses to the zero face in $\overline{Y_{0}}(\mathcal{A})$ ). Since vertices of $\overline{Y_{f}}(\mathcal{A})$ are f.m.p.t.'s and since all f.m.p.t.'s contain all the boundary edges and vertices, $Y_{f}(\mathcal{A})$ contains all the vertices of $\overline{Y_{f}}(\mathcal{A})$. Hence, its vertices are in bijection with all f.m.p.t.'s which, in turn, are in bijection with pseudo-triangulations. Edges of $Y_{f}(\mathcal{A})$ correspond to f.m.p.t.'s minus one interior edge or mark, which are precisely the flips between f.m.p.t.'s or between pseudotriangulations.

(2) The facets containing $F$ are those corresponding to marks in interior points. Then the faces in the complement of the star of $F$ are those in which none of the inequalities (5) are tight; that is to say, they form the poset of "non-crossing marked graphs containing 
the boundary edges and marks but no interior marks", which is the same as the poset of non-crossing graphs containing the boundary.

We now turn our attention to Theorem 1.4. Its proof is based on the use of the homogeneous cone $\overline{Y_{0}}(\mathcal{A})$ or, more preciesely, the set $\mathcal{H}:=\left\{H_{i j}: i, j=1, \ldots, n\right\} \cup$ $\left\{H_{0 i}: i=1, \ldots, n\right\}$ of hyperplanes that define it.

Proof of Theorem 1.4. Observe now that the equations defining $H_{i j}$, specialized to $t_{i}=0$ for every $i$, become the equations of the infinitesimal rigidity of the complete graph on $\mathcal{A}$. In particular, a graph $G$ is rigid on $\mathcal{A}$ if and only if the intersection

$$
\left(\bigcap_{i j \in G} H_{i j}\right) \cap\left(\bigcap_{i=1}^{n} H_{0 i}\right)
$$

equals zero.

This happens for any pseudo-triangulation because Theorem 3.9 implies that the hyperplanes corresponding to the $3 n-3$ edges and marks of any f.m.p.t. form a basis of the (dual of) the linear space $S$.

To prove part (2) we only need the fact that the $3 n-3$ linear hyperplanes corresponding to an f.m.p.t. are independent. In particular, any subset of them is independent too. We consider the subset corresponding to the induced (marked) subgraph on the $n-k-l$ vertices other than the $k$ pointed and $l$ non-pointed ones we are interested in. They form an independent set involving only $3(n-k-l)$ coordinates, hence their number is at most $3(n-k-l)-3$ (we need to subtract 3 for the rigid motions of the $n-k-l$ points, and here is where we need $k+l \leq n-2$ ). Since the f.m.p.t. has $3 n-3$ edges plus marks, at least $3 k+3 l$ of them are incident to our subset of points, and exactly $k$ marks are incident to our points, hence at least $2 k+3 l$ edges are.

Actually, we can derive some consequences for general planar rigid graphs. Observe that every planar and generically rigid graph $G$ must have between $2 n-3$ and $3 n-3$ edges (the extreme cases being an isostatic graph and a triangulation of the 2-sphere). Hence, we can say that the graph $G$ has $2 n-3+y$ edges, where $0 \leq y \leq n-3$. If the graph can be embedded as a pseudo-triangulation, then the embedding will have exactly $y$ non-pointed vertices. In particular, the following statement is an indication that every planar and rigid graph can be embedded as a pseudo-triangulation:

Proposition 3.11. Let $G$ be a planar and generically rigid graph with $n$ vertices and $2 n-3+y$ edges. Then there is a subset $Y$ of cardinality $y$ of the vertices of $G$ such that every set of $l$ vertices in $Y$ plus $k$ vertices not in $Y$ is incident to at least $2 k+3 l$ edges, whenever $k+l \leq n-2$.

Proof. Consider $G$ embedded planarly in a sufficiently generic straight-line manner. Since the embedding is planar, it can be completed to a pseudo-triangulation $T$. In particular, the set of edges of $G$ represents an independent subset of $2 n-3+y$ hyperplanes of $\mathcal{H}$. However, since the graph is rigid, adding marks to all the vertices produces a spanning set of $3 n-3+y$ hyperplanes. Between these two sets there must be a basis, 


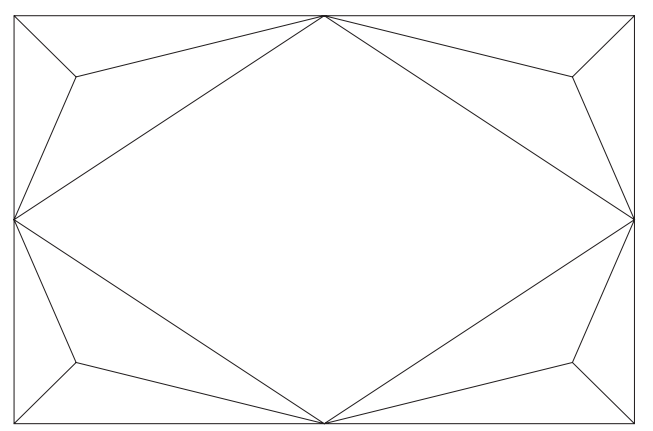

Fig. 7. A planar graph satisfying the conclusion of Proposition 3.11 need not be rigid

consisting of the $2 n-3+y$ edges of $G$ plus $n-y$ marks. We call $Y$ the vertices not marked in this basis, and the same argument as in the proof of Theorem 1.4 gives the statement.

It has to be said, however, that a planar graph $G$ with a subset $Y$ of its vertices satisfying Proposition 3.11 need not be generically rigid. Figure 7 shows an example (take as $Y$ any three of the four six-valent vertices).

\section{Valid Choices of $f$}

It remains to be proved that valid choices of parameters do exist. In particular, that the choice in Theorem 3.8 is valid. Our methods, again inspired by [19], actually give more: a full description of the set of valid choices via a set of $\left(\begin{array}{l}n \\ 4\end{array}\right)$ linear inequalities, one for each four-point subset of the $n$ points.

Definition 4.1. Let $G$ be a graph embedded on $\mathcal{A}$, with a set of edges $E$ and a set of marked vertices $V$. In our context, an equilibrium stress on $G$ (or a stress, for short) is an assignment of scalars $w_{i j}$ to edges and $\alpha_{j}$ to marked vertices of $G$, such that for every $(v, t) \in \mathbb{R}^{3 n}$,

$$
\sum_{i j \in E} w_{i j}\left(\left\langle p_{i}-p_{j}, v_{i}-v_{j}\right\rangle-\left|p_{i}-p_{j}\right|\left(t_{i}+t_{j}\right)\right)+\sum_{i \in V} \alpha_{i} t_{i}=0 .
$$

Lemma 4.2. Let $\sum_{i=1}^{n} \lambda_{i} p_{i}=0, \sum \lambda_{i}=0$, be an affine dependence on a point set $\mathcal{A}=\left\{p_{1}, \ldots, p_{n}\right\}$. Then

$$
w_{i j}:=\lambda_{i} \lambda_{j} \quad \text { for every } i, j
$$

and

$$
\alpha_{i}:=\sum_{j: i j \in E} \lambda_{i} \lambda_{j}\left|p_{i}-p_{j}\right| \quad \text { for every } i
$$

defines a stress of the complete graph $G$ on $\mathcal{A}$. 
Proof. Equation (6) on the variables $v$ gives

$$
\sum_{i j \in E} w_{i j}\left\langle p_{i}-p_{j}, v_{i}-v_{j}\right\rangle=0 \quad \text { for every } \quad v \in \mathbb{R}^{2 n} .
$$

This is fulfilled by the $w_{i j}$ 's of the statement

$$
\sum_{j \neq i} \lambda_{i} \lambda_{j}\left(p_{i}-p_{j}\right)=\sum_{j=1}^{n} \lambda_{i} \lambda_{j}\left(p_{i}-p_{j}\right)=\lambda_{i} p_{i} \sum_{j=1}^{n} \lambda_{j}-\lambda_{i} \sum_{j=1}^{n} \lambda_{j} p_{j}=0,
$$

where the last equality comes from the $\lambda_{i}$ 's being an affine dependence.

Then, in order for the coefficient of $t_{i}$ in (6) to cancel, we need $\alpha_{i}=\sum_{j: i j \in E} w_{i j} \mid p_{i}-$ $p_{j} \mid$.

Crucial for our construction is the case of four points in general position in $\mathbb{R}^{2}$. They have a unique (up to a constant) affine dependence, whose coefficients are

$$
\lambda_{i}=(-1)^{i} \operatorname{det}\left(\left[p_{1}, \ldots, p_{4}\right] \backslash\left\{p_{i}\right\}\right) .
$$

Dividing the self-stress of Lemma 4.2 by the constant

$$
-\operatorname{det}\left(p_{1}, p_{2}, p_{3}\right) \operatorname{det}\left(p_{1}, p_{2}, p_{4}\right) \operatorname{det}\left(p_{1}, p_{3}, p_{4}\right) \operatorname{det}\left(p_{2}, p_{3}, p_{4}\right)
$$

we derive that the following expressions form a self-stress of the complete marked graph on these four points:

$$
w_{i j}=\frac{1}{\operatorname{det}\left(p_{i}, p_{j}, p_{k}\right) \operatorname{det}\left(p_{i}, p_{j}, p_{l}\right)}, \quad \alpha_{i}=\sum_{j: i j \in E} w_{i j}\left|p_{i}-p_{j}\right| .
$$

In the equation for $w_{i j}, k$ and $l$ denote the two indices other than $i$ and $j$. The reason for not taking the self-stress of Lemma 4.2 directly is that our normalization of it produces the following key property which turns out to be fundamental later; see Fig. 8:

Lemma 4.3. For any four points in general position, expressions (8) make $w_{i j}$ and $\alpha_{j}$ positive for boundary edges and points and negative for interior edges and points.
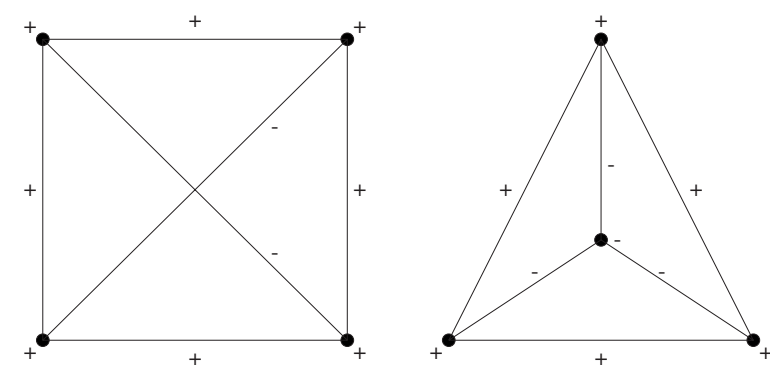

Fig. 8. The negative parts of these two marked graphs are the excluded minors in non-crossing marked graphs of a point set in general position. 
Proof. In order to check the part concerning $w_{i j}$ 's we use that $\operatorname{det}\left(q_{1}, q_{2}, q_{3}\right)$ is twice the signed area of the triangle spanned by $q_{1}, q_{2}, q_{3}$ : For a boundary edge the two remaining points lie on the same side of the edge, so they have the same sign. For an interior edge, they lie on opposite sides and therefore they have different signs.

For the $\alpha_{i}$ 's, if $i$ is an interior point, then all the $w_{i, j}$ 's in the formula for $\alpha_{i}$ are negative and, hence, $\alpha_{i}$ is also negative. If $i$ is a boundary point, then two of the $w_{i, j}$ are positive and the third one is negative. However, since

$$
\sum_{j \in\{1,2,3,4\} \backslash i} w_{i, j}\left(p_{i}-p_{j}\right)=0,
$$

the triangle inequality implies that the two positive summands $w_{i j}\left|p_{i}-p_{j}\right|$ in the expression of $\alpha_{i}$ add up to a greater absolute value than the negative one. Hence, $\alpha_{i}$ is positive.

The previous statement is crucial to us, because no matter whether the four points are in convex position or one of them is inside the convex hull of the other three, the f.m.p.t.'s on the four points can be characterized as the marked graphs with nine edges plus marks and in which the missing edge or mark is interior (two f.m.p.t's for points in convex position, four of them for a triangle plus an interior point).

We conclude that:

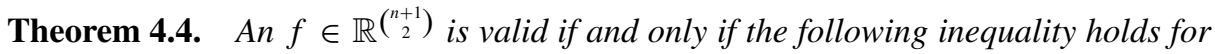
every four points $\left\{p_{1}, p_{2}, p_{3}, p_{4}\right\}$ of $\mathcal{A}$ :

$$
\sum_{1 \leq i<j \leq 4} w_{i j} f_{i j}+\sum_{j=1}^{4} \alpha_{j} f_{0 j}>0,
$$

where the $w_{i j}$ 's and $\alpha_{j}$ 's are those of (8).

Proof. Suppose first that $\mathcal{A}$ has only four points. The polyhedron $\overline{Y_{f}}(\mathcal{A})$ is ninedimensional, which implies that for every vertex $(v, t)$ of the polyhedron, the set $T(v, t)$ contains at least nine edges plus marks on those four points. Therefore, $T(v, t)$ is the complete marked graph with an edge or mark removed.

We denote by $G_{k}$ and $G_{k l}$ the complete marked graph with a non-marked vertex $k$ or a missing edge $k l$, respectively. Recall that by Lemma 4.3 the choice of stress on four points has the property that $G_{k}$ and $G_{k l}$ are f.m.p.t.'s if and only if $\alpha_{k}$ and $w_{k l}$ (corresponding respectively to the removed mark or edge) are negative. Let us see that this is equivalent to $f$ being valid:

By the definition of stress,

$$
\sum_{1 \leq i<j \leq 4} w_{i j}\left(\left\langle p_{i}-p_{j}, v_{i}-v_{j}\right\rangle-\left|p_{i}-p_{j}\right|\left(t_{i}+t_{j}\right)\right)+\sum_{j=1}^{4} \alpha_{j} t_{j}
$$

equals zero. In the case of $G_{k}$, in which every edge and vertex except $k$ are tight, that 
expression equals

$$
\sum_{1 \leq i<j \leq 4} w_{i j} f_{i j}+\sum_{j=1}^{4} \alpha_{j} f_{0 j}+\alpha_{k}\left(t_{k}-f_{0 k}\right) .
$$

In the case of $G_{k l}$, where every vertex and edge except $k l$ are tight, it equals

$$
\sum_{1 \leq i<j \leq 4} w_{i j} f_{i j}+\sum_{j=1}^{4} \alpha_{j} f_{0 j}+w_{k l}\left(\left\langle p_{k}-p_{l}, v_{k}-v_{l}\right\rangle-\left|p_{k}-p_{l}\right|\left(t_{k}+t_{l}\right)-f_{k l}\right) .
$$

Since $\left\langle p_{k}-p_{l}, v_{k}-v_{l}\right\rangle-\left|p_{k}-p_{l}\right|\left(t_{k}+t_{l}\right)-f_{k l} \geq 0$ and $t_{k}-f_{0 k} \geq 0$, by (4) and (5), we conclude that in the first and second cases above, $\alpha_{k}$ and $w_{k l}$, respectively, are negative if and only if $f$ is valid.

Now we turn to the case of a general $\mathcal{A}$ and our task is to prove that a choice of parameters $f$ is valid if and only if it is valid when restricted to any four points. Observe that if $\mathcal{A}^{\prime} \subset \mathcal{A}$, then $Y_{f}\left(\mathcal{A}^{\prime}\right)$ equals the intersection of $Y_{f}(\mathcal{A})$ with the subspace where $v_{i}=0$ and $t_{i}=0$ for all $p_{i} \in \mathcal{A} \backslash \mathcal{A}^{\prime}$. In particular, the marked graphs on $\mathcal{A}^{\prime}$ corresponding to faces of $Y_{f}\left(\mathcal{A}^{\prime}\right)$ are subgraphs of marked graphs of faces of $Y_{f}(\mathcal{A})$. Moreover, noncrossingness of a marked graph on $\mathcal{A}$ is equivalent to non-crossingness of every induced marked graph on four vertices: indeed, a crossing of two edges appears in the marked graph induced by the four endpoints of the two edges, and a non-pointed marked vertex appears in the marked graph induced on the four endpoints involved in any three edges forming a non-pointed "letter Y" at the non-pointed vertex.

Hence, if $f$ is valid for every four points, then none of the four-point minors forbidden by non-crossingness appear in faces of $Y_{f}(\mathcal{A})$ and $f$ is valid for $\mathcal{A}$. Conversely, if $f$ is not valid on some four-point subset $\mathcal{A}^{\prime}$, then the marked graph on $\mathcal{A}^{\prime}$ corresponding to any vertex of $Y_{f}\left(\mathcal{A}^{\prime}\right)$ would be the complete graph minus one boundary edge or vertex, that is to say, it would not be non-crossing. Hence $f$ would not be valid on $\mathcal{A}$ either.

Corollary 4.5. For any $a, b \in \mathbb{R}^{2}$, the choice $f_{i j}:=\operatorname{det}\left(a, p_{i}, p_{j}\right) \operatorname{det}\left(b, p_{i}, p_{j}\right)$, $f_{0 j}:=0$ is valid.

Proof. Consider the four points $p_{i}$ as fixed and regard $R:=\sum w_{i j} f_{i j}+\sum \alpha_{j} f_{0 j}=$ $\sum w_{i j} f_{i j}$ as a function of $a$ and $b$ :

$$
R(a, b)=\sum_{1 \leq i<j \leq 4} \operatorname{det}\left(a, p_{i}, p_{j}\right) \operatorname{det}\left(b, p_{i}, p_{j}\right) w_{i j} .
$$

We have to show that $R(a, b)$ is always positive. We actually claim it to be always one. Observe first that $R\left(p_{i}, p_{j}\right)$ is trivially one for $i \neq j$. Since any three of our points are an affine basis and since $R(a, b)$ is an affine function of $b$ for fixed $a$, we conclude that $R\left(p_{i}, b\right)$ is one for every $i \in\{1,2,3,4\}$ and for every $b$. The same argument shows that $R(a, b)$ is constantly one: for fixed $b$ it is an affine function of $a$ and is equal to one on an affine basis. 


\section{Points in Special Position}

In this section we show that almost everything we said so far applies equally to point sets with collinear points. We essentially follow the same steps as in Sections 2-4. Two subtleties are that our definitions of pointedness or pseudo-triangulations can only be fully justified a posteriori, and that the construction of the polyhedron for point sets with boundary collinearities is slightly indirect: it relies on the choice of some extra exterior points to make colliniarities go to the interior.

\section{The Graph of All Pseudo-Triangulations of $\mathcal{A}$}

Definition 5.1. Let $\mathcal{A}$ be a point set in the plane, possibly with collinear points.

(1) A graph $G$ with a vertex set $\mathcal{A}$ is called non-crossing if no edge intersects another edge of $G$ or a point of $\mathcal{A}$ except at its endpoints. In particular, if $p_{1}, p_{2}$ and $p_{3}$ are three collinear points, in this order, then the edge $p_{1} p_{3}$ cannot appear in a non-crossing graph, independently of whether there is an edge incident to $p_{2}$ or not.

(2) A pseudo-triangle is a simple polygon with only three interior angles smaller than $\pi$. A pseudo-triangulation of $\mathcal{A}$ is a non-crossing graph with vertex set $\mathcal{A}$, which partitions $\operatorname{conv}(\mathcal{A})$ into pseudo-triangles and such that no point in the interior of $\operatorname{conv}(\mathcal{A})$ is incident to more than one angle of $\pi$.

Figure 9 shows the eight pseudo-triangulations of a certain point set. We have drawn them connected by certain flips, to be defined later, and with certain points marked. The graph on the right of the figure is not a pseudo-triangulation because it fails to satisfy
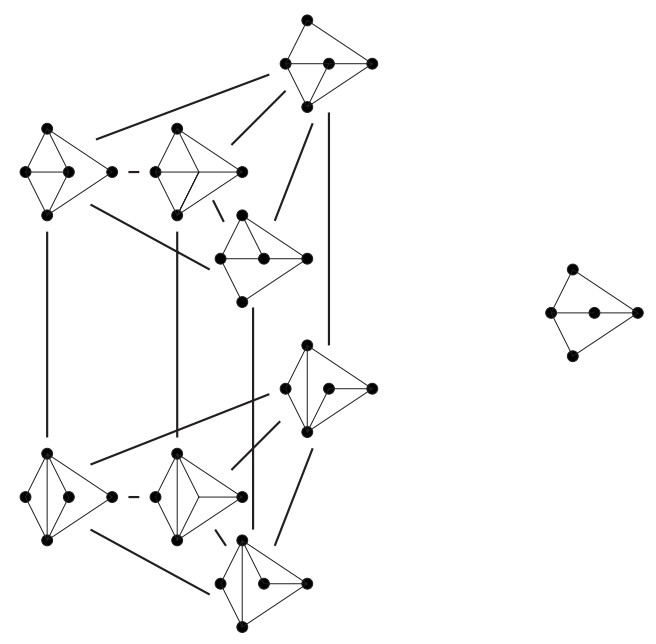

Fig. 9. The eight pseudo-triangulations of a point set with interior collinearities (left) plus a non-crossing graph with pseudo-triangular faces but which we do not consider a pseudo-triangulation (right). 


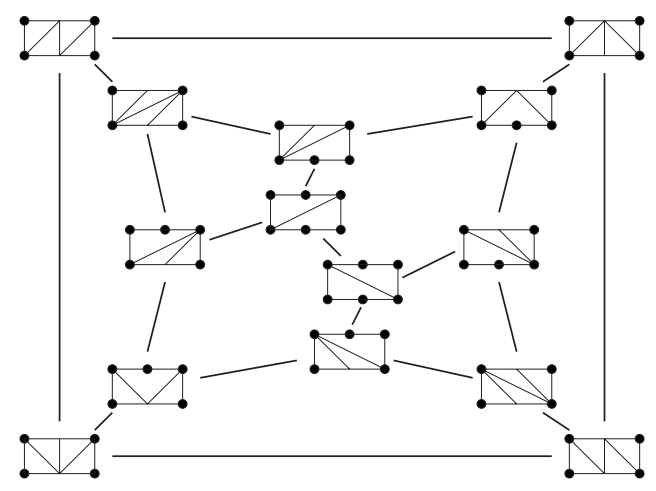

Fig. 10. The graph of pseudo-triangulations of a point set with boundary collinearities.

the last condition in our definition. Intuitively, the reason why we do not allow it as a pseudo-triangulation is that we are considering angles of exactly $\pi$ as being reflex, and we do not want a vertex to be incident to two reflex angles.

However, if collinearities happen in the boundary of $\operatorname{conv}(\mathcal{A})$, as in Fig. 10, we treat things differently. The exterior angle of $\pi$ is not counted as reflex, and hence the middle point in a boundary collinearity is allowed to be incident to an interior angle of $\pi$. This choice of convention can only be fully justified "a posteriori": it is the one that produces the result we are interested in (Corollary 5.16). For the impatient reader, maybe the following argument will suffice: we certainly do not want an interior vertex to be incident to two angles of $\pi$, because then there would be two different ways to insert an edge incident to that vertex, and the graph of flips would necessarily be non-regular (see Fig. 9 again). At a semi-interior vertex this is not an issue and our convention actually produces a much better behaved graph of flips than the one not allowing $\pi$ interior angles at semi-interior vertices (compare Figs. 10 and 15).

Definition 5.2. A vertex $p$ in a non-crossing graph on $\mathcal{A}$ is considered pointed if either (1) it is a vertex of $\operatorname{conv}(\mathcal{A}),(2)$ it is semi-interior and not incident to any edge going through the interior of $\operatorname{conv}(\mathcal{A})$ or $(3)$ it is interior and its incident edges span at most $\pi$ radians.

A non-crossing marked graph is a non-crossing graph with marks at some of its pointed vertices. If all pointed vertices are marked we say the non-crossing graph is fully marked. Marks at interior and semi-interior points are called interior marks.

For example, all the graphs of Figs. 9 and 10 are fully marked. That is to say, big dots correspond exactly to pointed vertices. Of course, f.m.p.t.'s are just pseudo-triangulations with marks at all their pointed vertices. Observe that we are calling interior marks and edges exactly those which do not appear in all pseudo-triangulations. From now on, we denote by $n_{i}, n_{s}$ and $n_{v}$ the number of interior, semi-interior and extremal points of $\mathcal{A}$. Finally, $n=n_{v}+n_{s}+n_{i}$ denotes the total number of points in $\mathcal{A}$. The following two statements essentially say that Proposition 2.9 is valid for non-generic configurations. 
Lemma 5.3. F.m.p.t.'s are exactly the maximal marked non-crossing graphs on $\mathcal{A}$. They all have $3 n-n_{s}-3$ edges plus marks and $2 n_{i}+n-3$ interior edges plus interior marks.

Proof. The first sentence is equivalent to saying that every non-crossing graph $G$ can be completed to a pseudo-triangulation without making any pointed vertex non-pointed. The proof of this is that if $G$ is not a pseudo-triangulation, then either it has a face with more than three corners, in which case we insert the diagonal coming from the geodesic between any two non-adjacent corners, or there is an interior vertex with two angles of $\pi$, in which case we choose to consider one of them as reflex and the other as convex, and insert the diagonal joining the convex angle to the opposite corner of the pseudo-triangle containing it.

To prove the cardinality of pseudo-triangulations, let $n_{\varepsilon}$ denote the number of marks. Let us think of boundary collinearities as if they were concave boundary chains in our graph, and triangulate the polygons formed by these chains by adding (combinatorially or topologically) $n_{s}$ edges in total. If, in addition, we consider interior angles of $\pi$ or more as reflex and the others as convex, we get a graph with all the combinatorial properties of pseudo-triangulations and, in particular, a graph for which Proposition 2.2 can be applied, since its proof is purely combinatorial (a double counting of convex angles, combined with Euler's relation). In particular, the extended graph has $3 n-3$ edges plus marks, and the original graph has $3 n-3-n_{s}$ of them. Since there are exactly $n_{s}+n_{v}$ exterior edges and $n_{v}$ exterior marks in every pseudo-triangulation, the last sentence follows.

Lemma 5.4. If an interior edge or mark is removed from an f.m.p.t., then there is a unique way to insert another edge or mark to obtain a different f.m.p.t.

Proof. If an edge is removed, then there are three possibilities: (1) The removal does not create any new reflex angle, in which case the region obtained by the removal is a pseudo-quadrangle (that is, it has four non-reflex angles), because the two regions incident to it had six corners in total and the number of them decreases by two. We insert the opposite diagonal of it. (2) The removal creates a new reflex angle at a vertex which was not pointed. Then the region obtained is a pseudo-triangle and we just add a mark at the new pointed vertex. (3) The removal creates a new reflex angle at a vertex that was already pointed. This means that after the removal the vertex has two reflex angles, that is to say two angles of exactly $\pi$ each. We insert the edge joining this vertex to the opposite corner of the pseudo-triangle containing the original reflex angle.

If a mark is removed, then the only possibility is: (4) The pointed vertex holding the mark is incident to a unique reflex angle (remember that we consider interior angles of $\pi$ as reflex). We insert the edge joining the vertex to the opposite corner of the corresponding pseudo-triangle.

Definition 5.5. Two f.m.p.t.'s are said to differ by a flip if they differ by just one edge or mark. Cases (1)-(4) in the previous proof are called, respectively, diagonal flip, deletion flip, mirror flip and insertion flip. 
Of course, our definition of flips specializes to the one for points in general position, except that mirror flips can only appear in the presence of collinearities. An example of a mirror flip can be seen towards the upper right corner of Fig. 9.

Corollary 5.6. The graph of flips between f.m.p.t.'s of a planar point set is connected and regular of degree $2 n_{i}+n-3$.

The reader will have noticed that the graphs of Figs. 9 and 10 are more than regular of degrees 4 and 3, respectively. They are the graphs of certain simple polytopes of dimensions 4 and 3. (Fig. 9 is a prism over a simplex.)

The Case with Only Interior Collinearities. Now we assume that our point set $\mathcal{A}$ has only interior collinearities.

For each $f \in \mathbb{R}^{n+1}$ let $\overline{Y_{f}}(\mathcal{A})$ be the polyhedron defined in Section 3. Recall that everything we said in that section, up to Corollary 3.6, is valid for points in special position. Our main result here is that Theorems 3.8 and 3.9 hold word by word in the case with no boundary collinearities, except that precision needs to be made regarding the concept of validity.

Recall that for a given choice of $f \in \mathbb{R}^{\left(\begin{array}{c}n+1 \\ 2\end{array}\right)}$, an edge $p_{i} p_{j}$ or a point $p_{i}$ is called tight for a certain $(v, t) \in \mathbb{R}^{3 n-3}$ or for a face $F$ of $\overline{Y_{f}}(\mathcal{A})$ if the corresponding equation (4) or (5) is satisfied with equality.

Definition 5.7. For any $(v, t) \in \mathbb{R}^{3 n-3}$, we denote by $T(v, t)$ the marked graph of all its tight edges and points. We call it a strict supporting graph of $(v, t)$. We call the weak supporting graph of a $(v, t)$ the marked subgraph consisting of edges and points of $T(v, t)$ which define facets of $\overline{Y_{f}}(\mathcal{A})$.

We call the (strict or weak) supporting graph of a face $F$ of $\overline{Y_{f}}(\mathcal{A})$ one of any point $(v, t)$ in the relative interior of $F$.

A choice of $f$ is called weakly valid (resp., strictly valid) if the weak (resp., strict) supporting graphs of all the faces of $\overline{Y_{f}}(\mathcal{A})$ are non-crossing marked graphs.

Observe that from any weakly valid choice $f$ one can obtain strictly valid ones: just decrease by arbitrary positive amounts the coordinates of $f$ corresponding to equations which do not define facets of $\overline{Y_{f}}(\mathcal{A})$. Hence, we could do what follows only in terms of strict validity and would obtain the same polyhedron. However, weak validity is needed, as we will see in Remark 5.13, if we want our construction to depend continuously on the coordinates of the point set $\mathcal{A}$.

To obtain the equations that valid choices must satisfy we proceed as in Section 4. The crucial point there was that a marked graph is non-crossing if and only if it does not contain the negative parts of the unique stress in certain subgraphs.

Lemma 5.8. Let $\mathcal{A}$ be a point set with no three collinear boundary points. Then a marked graph on $\mathcal{A}$ is non-crossing if and only if it does not contain any of the following four marked subgraphs: the negative parts of the marked graphs displayed in Fig. 8 and the negative parts of the marked graphs displayed in Fig. 11. 

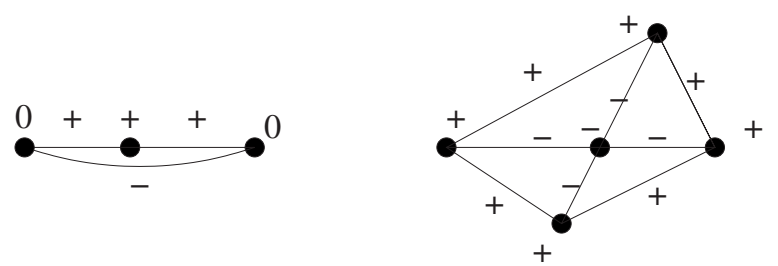

Fig. 11. The two additional excluded minors for non-crossing marked graphs of a point set with interior collinearities.

Proof. Exclusion of the negative parts of the left graphs in both figures are our definition of crossingness for an unmarked graph. An interior vertex is pointed if and only if none of the negative parts of the right graphs appear.

Lemma 5.9. The two graphs in Fig. 11 have a stress with signs as in the figure.

Proof. For the left part it is easy to show that the following is a stress:

$$
\begin{aligned}
w_{12} & =\frac{1}{\left|p_{2}-p_{1}\right|}, \quad w_{13}=-\frac{1}{\left|p_{3}-p_{1}\right|}, \quad w_{23}=\frac{1}{\left|p_{3}-p_{2}\right|}, \\
\alpha_{1} & =\alpha_{3}=0, \quad \alpha_{2}=2 .
\end{aligned}
$$

For the right part, observe that, by definition, stresses on a marked graph form a linear space. Let the four exterior points be $p_{1}, p_{2}, p_{3}$ and $p_{4}$, in cyclic order, and let the interior point be $p_{5}$. We know three different stresses of the complete graph on these five points: the one we used in Section 4 for the four exterior points and the two that we have just introduced for the two collinear triplets. From these three we can eliminate the coordinates of edges $p_{1} p_{3}$ and $p_{2} p_{4}$ and we get a stress with the stated signs.

Theorem 5.10. Let $\mathcal{A}$ be a point set with no three collinear boundary points. Then a choice of $f$ is weakly valid if it satisfies (9) for all quadruples of points in general position plus the following sets of equations:

- For any three points $p_{1}, p_{2}$ and $p_{3}$ collinear in this order,

$$
\frac{f_{12}}{\left|p_{2}-p_{1}\right|}-\frac{f_{13}}{\left|p_{3}-p_{1}\right|}+\frac{f_{23}}{\left|p_{3}-p_{1}\right|}+2 f_{02} \geq 0
$$

- For any five points as in the right part of Fig. 11, the following equation where the $w_{i j}$ 's and the $\alpha_{i}$ 's form a stress with signs as indicated in the figure (by convention, $w_{i j}$ equals zero for the two missing edges in the graph):

$$
\sum_{1 \leq i<j \leq 5} w_{i j} f_{i j}+\sum_{j=1}^{5} \alpha_{j} f_{0 j}>0
$$

The choice is strictly valid if and only if, moreover, equation (10) of every collinear triplet is satisfied strictly. 
Proof. Equations (9) guarantee that no weak or strict tight graph contains the two excluded marked graphs of negative edges and points of Fig. 8. Equations (10) and (11) with strict inequality, do the same for the graphs of Fig. 11. That these equations are equivalent to strict validity is proved exactly as in Section 4 . The reason why we allow equality in (10) if we only want a weakly valid choice is that the negative part of the stress consists of a single edge. If the equation is satisfied with equality, then the hyperplane corresponding to this edge is a supporting hyperplane of the face of $\overline{Y_{f}}$ given by the intersection of the three hyperplanes of the positive part of the stress.

Corollary 5.11. Let $\mathcal{A}$ be a point set with no collinear boundary points. Any choice of $f$ satisfying (9) for every four points in general position plus the following ones for every collinear triplet is weakly valid:

$$
\frac{f_{12}}{\left|p_{2}-p_{1}\right|}-\frac{f_{13}}{\left|p_{3}-p_{1}\right|}+\frac{f_{23}}{\left|p_{3}-p_{1}\right|}+2 f_{02}=0 .
$$

In particular, the choices of Corollary 4.5 and Theorem 3.8 are weakly valid.

Proof. For the first assertion, we need to show that (11) follows from (9) and (12). However, this is straightforward: from our proof of Lemma 5.9 it follows that (11) is just the equation obtained by substituting in (9) the values for $w_{13}$ and $w_{24}$ obtained from (12).

For the last assertion, we already proved in Corollary 4.5 that the choices of $f$ introduced there satisfy (9). It is easy, and left to the reader, to show that they also satisfy (12).

Theorem 5.12 (Main Theorem, Case without Boundary Collinearities). Let $\mathcal{A}$ be a point set with no three collinear points in the boundary of $\operatorname{conv}(\mathcal{A})$, and let $f$ be a weakly valid choice of parameters. Then:

(1) $\overline{Y_{f}}$ is a simple polyhedron of dimension $3 n-3$ whose face poset equals (the opposite of) the poset of non-crossing marked graphs on $\mathcal{A}$.

(2) The face $Y_{f}$ defined by turning into equalities equations (4) and (5) corresponding to boundary vertices and edges is the unique maximal bounded face of $\overline{Y_{f}}$. It is a simple polytope of dimension $2 n_{i}+n-3$ whose 1-skeleton is the graph of pseudo-triangulations of $\mathcal{A}$.

(3) Let $F$ be the face of $Y_{f}$ defined by turning into equalities the remaining equations (5). The complement of the star of $F$ in the face-poset of $Y_{f}$ equals the poset of non-crossing graphs on $\mathcal{A}$ that use all the convex hull edges.

Proof. Recall that if no three boundary points are collinear, then every f.m.p.t. (i.e., maximal marked non-crossing graph) has $3 n-3$ edges plus marks, exactly as in the general position case (Lemma 5.3). In particular, it is still true, for the same reasons as in the general position case, that $\overline{Y_{f}}(\mathcal{A})$ is simple and all its vertices correspond to f.m.p.t.'s, for any valid choice of $f$. The rest of the arguments in the proof of Theorem 3.9 rely on the graph of flips being connected, a property that we still have. As for 

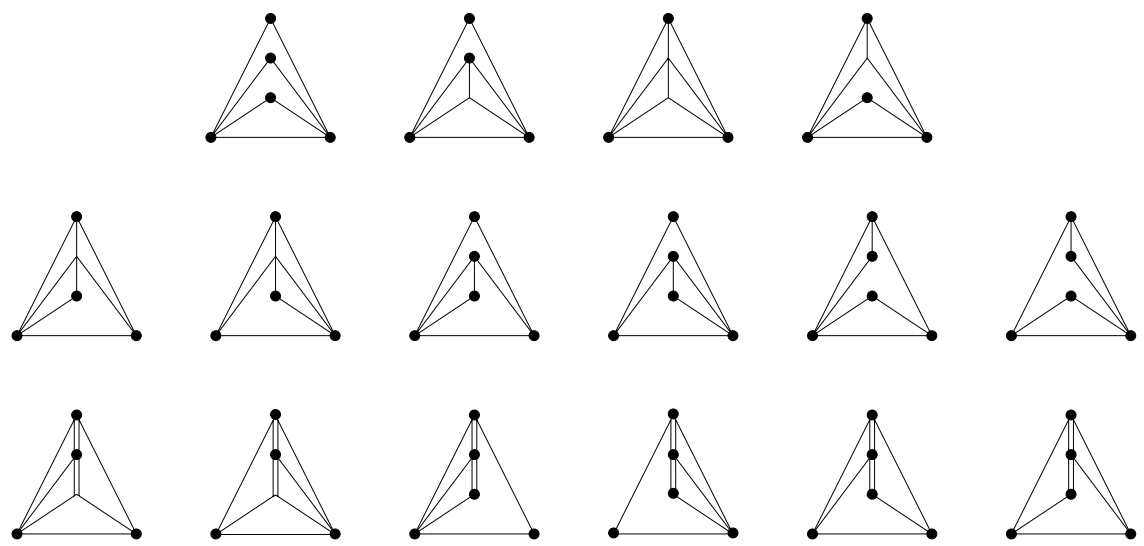

Fig. 12. The 16 pseudo-triangulations of $\mathcal{A}_{1}$.

Theorem 3.10, the face $Y_{f}(\mathcal{A})$ is bounded because Lemma 3.4 still applies. The rest is straightforward.

Remark 5.13. It is interesting to observe that taking the explicit valid choice of $f$ of Theorem 3.8 the equations defining $\overline{Y_{f}}(\mathcal{A})$ depend continuously on the coordinates of the points in $\mathcal{A}$. When three points become collinear, the hyperplane corresponding to the (now) forbidden edge becomes, as we said in the proof of Theorem 5.10, a supporting hyperplane of a codimension 3 face of $\overline{Y_{f}}(\mathcal{A})$. The combinatorics of the polytope changes but maintaining its simplicity. This continuity of the defining hyperplanes would clearly be impossible if we required our choice to be strictly valid for point sets with collinearities.

Example 5.14. Let $\mathcal{A}_{1}$ and $\mathcal{A}_{2}$ be the two point sets with five points each whose pseudo-triangulations are depicted in Figs. 12 and 13. The first one has three collinear points and the second is obtained by perturbation of the collinearity. These two examples were computed with the software $\mathrm{CDD}+$ of Fukuda [9] before we had a clear idea of what the right definition of a pseudo-triangulation for points in special position should be. To emphasize the meaning of weak validity, in Fig. 12 we show the weak supporting graphs of the vertices of $\overline{Y_{f}}\left(\mathcal{A}_{1}\right)$, rather than the strict ones.

The first ten pseudo-triangulations are common to both figures (upper two rows). Only the pseudo-triangulations of $\mathcal{A}_{1}$ using the two collinear edges plus the mark at the central point of the collinearity are affected by the perturbation of the point set. This is no surprise since these two edges plus this mark are the positive part of the stress involved in the collinearity. At each of these six pseudo-triangulations, the hyperplane of the big edge is tangent to the vertex of $\overline{Y_{f}}\left(\mathcal{A}_{1}\right)$ corresponding to the pseudo-triangulation. When the collinearity is perturbed, this hyperplane moves in one of the two possible ways: away from the polyhedron, in which case the combinatorics is not changed, or towards the interior of the polyhedron, in which case the old vertex disappears and some new vertices are cut by this hyperplane. In our case, these two behaviors appear each in three 

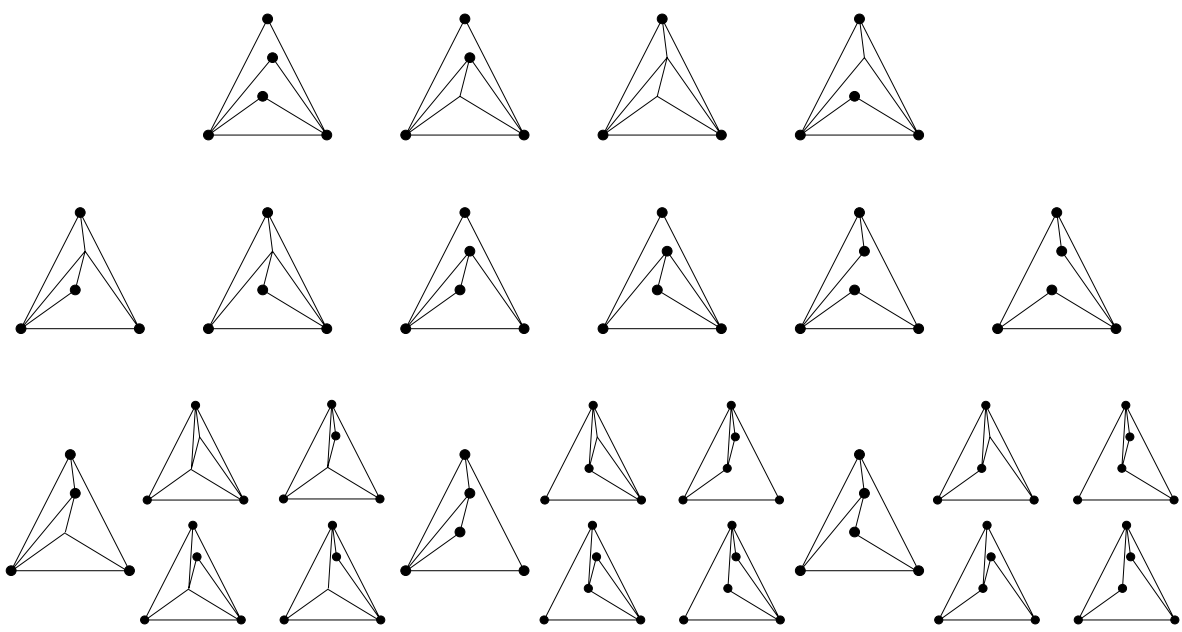

Fig. 13. The 25 pseudo-triangulations of $\mathcal{A}_{2}$.

of the six "non-strict" pseudo-triangulations of $\mathcal{A}_{1}$. When the hyperplane moves towards the interior, four new vertices appear where there was one.

Boundary Collinearities. In the presence of boundary collinearities, Lemma 5.3 implies a significant difference: with the equations we have used so far, the face of $\overline{Y_{0}}(\mathcal{A})$ defined by tightness at boundary edges and vertices is not the origin, but an unbounded cone of dimension $n_{s}$. Indeed, for each semi-interior point $p_{i}$, the vector $(v, t)$ with $v_{i}$ an exterior normal to the boundary of $\operatorname{conv}(\mathcal{A})$ at $p_{i}$ and every other coordinate equal to zero defines an extremal ray of that face. As a consequence, the corresponding face in $\overline{Y_{f}}(\mathcal{A})$ is unbounded.

We believe that it should be possible to obtain a polyhedron with the properties we want by just intersecting the polyhedron of our general definition with $n_{s}$ hyperplanes. However, instead of doing this we use the following simple trick to reduce this case to the previous one. From a point set $\mathcal{A}$ with boundary collinearities we construct another point set $\mathcal{A}^{\prime}$ adding to $\mathcal{A}$ one point in the exterior of each edge of $\operatorname{conv}(\mathcal{A})$ that contains semi-interior points (Fig. 14).

Lemma 5.15. A marked graph $G$ on $\mathcal{A}$ is non-crossing if and only if it becomes a non-crossing graph on $\mathcal{A}^{\prime}$ when we add to it the marks on all points of $\mathcal{A}^{\prime} \backslash \mathcal{A}$ and the
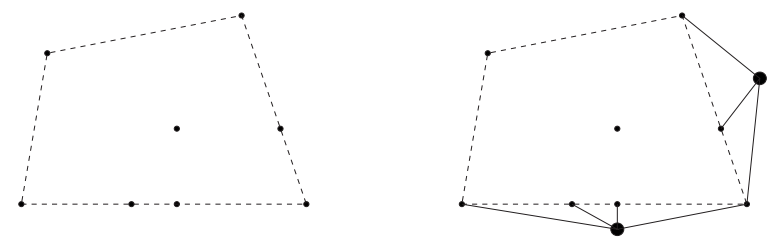

Fig. 14. The extended point set $\mathcal{A}^{\prime}$ and the relation between non-crossing marked graphs on $\mathcal{A}$ and $\mathcal{A}^{\prime}$. 
edges connecting each of these points to all the points of $\mathcal{A}$ lying in the corresponding edge of $\operatorname{conv}(\mathcal{A})$.

Proof. Straightforward.

In particular, we can construct the polyhedron $\overline{Y_{f}}\left(\mathcal{A}^{\prime}\right)$ for this extended point set $\mathcal{A}^{\prime}$ (taking any $f$ valid on $\mathcal{A}^{\prime}$ ), and call $\overline{Y_{f}}(\mathcal{A})$ the face of $\overline{Y_{f}}\left(\mathcal{A}^{\prime}\right)$ corresponding to the edges and marks mentioned in the statement of Lemma 5.15. Then:

Corollary 5.16 (Main Theorem, Case with Boundary Collinearities). In these conditions:

(1) $\overline{Y_{f}}$ is a simple polyhedron of dimension $3 n-3-n_{s}$ whose face poset equals (the opposite of) the poset of non-crossing marked graphs on $\mathcal{A}$.

(2) The face $Y_{f}$ defined by turning into equalities equations (4) corresponding to the $n_{v}+n_{s}$ edges between consecutive boundary points and equations (5) for the $n_{v}$ marks at vertices of $\operatorname{conv}(\mathcal{A})$ is the unique maximal bounded face of $\overline{Y_{f}}$. It is a simple polytope of dimension $2 n_{i}+n-3$ whose 1-skeleton is the graph of pseudo-triangulations of $\mathcal{A}$.

(3) Let $F$ be the face of $Y_{f}$ defined by turning into equalities the remaining $n-n_{v}$ equations (5). The complement of the star of $F$ in the face-poset of $Y_{f}$ equals the poset of non-crossing graphs on $\mathcal{A}$ that use all the convex hull edges.

Remark 5.17. The reader may wonder about the combinatorics of the polyhedron $\overline{Y_{f}}(\mathcal{A})$ that one would obtain with the equations of the generic case. Clearly, the tight graphs of its faces will not contain any of the four forbidden subgraphs of Lemma 5.8. It can be checked that the maximal marked graphs without those subgraphs all have $3 n-3$ edges plus marks and have the following characterization: as graphs they are pseudotriangulations in which all the semi-interior vertices are incident to interior edges, and they have marks at all the boundary points and at the pointed interior points. In other words, they would be the f.m.p.t.'s if we treated semi-interior points exactly as interior ones, hence forbidding them to be incident to two angles of $\pi$ and considering them always pointed since they are incident to one angle of $\pi$.

For example, in the point set of Fig. 10 there are six such graphs, namely the ones shown in Fig. 15.

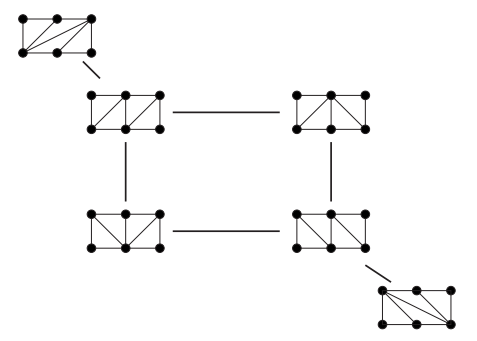

Fig. 15. The bounded part of $\overline{Y_{f}}(\mathcal{A})$ for a point set with boundary collinearities. 
This implies that the polyhedron $\overline{Y_{f}}(\mathcal{A})$ is still simple. The reason why we prefer the definitions we have given is that the polyhedron no longer has a unique maximal bounded face (it has three in the example of Fig. 15) and the graph of flips is no longer regular.

Observe finally that the proof of Theorem 1.4 given at the end of Section 3 is valid for points in special position, without much change: in all cases the hyperplanes corresponding to the edges of a pseudo-triangulation are independent in $\overline{Y_{0}}(\mathcal{A})$.

\section{References}

1. P. Agarwal, J. Basch, L. Guibas, J. Hershberger, and L. Zhang. Deformable free space tilings for kinetic collision detection. In B. R. Donald, K. Lynch, and D. Rus, editors, Algorithmic and Computational Robotics: New Directions, 4th International Workshop on Algebraic Foundations of Robotics (WAFR), pages 83-96. A. K. Peters, Boston, MA, 2000.

2. O. Aichholzer, F. Aurenhammer, P. Brass, and H. Krasser. Pseudo-triangulations from surfaces and a novel type of flip. SIAM J. Comput., 32 (2003), 1621-1653.

3. O. Aichholzer, F. Aurenhammer, and H. Krasser. Adapting (pseudo)-triangulations with a near-linear number of edge flips. In Proc. 8th International Workshop on Algorithms and Data Structures (WADS), pp. 12-24. Lecture Notes in Computer Science, volume 2748. Springer-Verlag, Berlin, 2003.

4. S. Bespamyatnikh. Transforming pseudo-triangulations. Inform. Process. Lett., 90(3) (2004), 141-145.

5. L. J. Billera, P. Filliman, and B. Sturmfels. Constructions and complexity of secondary polytopes. Adv. Math., 83 (1990), 155-179.

6. R. Connelly, E. Demaine, and G. Rote. Straightening polygonal arcs and convexifying polygonal cycles. In Proc. 41st Annual Symposium on Foundations of Computer Science (FOCS), Redondo Beach, California, pp. 432-452, 2000.

7. R. Connelly, E. D. Demaine, and G. Rote. Blowing up polygonal linkages. Discrete Comput. Geom., 30(2), (2003), 205-239.

8. J. A. de Loera, S. Hoşten, F. Santos, and B. Sturmfels. The polytope of all triangulations of a point configuration. Doc. Math., 1 (1996), 103-119.

9. K. Fukuda. Software available at http://www.cs.mcgill.ca/ fukuda/soft/cdd_home/cdd. html.

10. M. Goodrich and R. Tamassia. Dynamic ray shooting and shortest paths in planar subdivisions via balanced geodesic triangulations. J. Algorithms, 23 (1997), 51-73.

11. J. Graver, B. Servatius, and H. Servatius. Combinatorial Rigidity. Graduate Studies in Mathematics, volume 2. American Mathematical Society, Providence, RI, 1993.

12. R. Haas, D. Orden, G. Rote, F. Santos, B. Servatius, H. Servatius, D. Souvaine, I. Streinu and W. Whiteley. Planar minimally rigid graphs and pseudo-triangulations. Comput. Geom., to appear. Preliminary version in S. Fortune, editor, Proc. Nineteenth Conference on Computational Geometry, San Diego, California, pp. 154-163. ACM Press, New York, 2003.

13. D. Kirkpatrick, J. Snoeyink, and B. Speckmann. Kinetic collision detection for simple polygons. Internat. J. Comput. Geom. Appl., 12 (2002), 3-27.

14. C. Lee. The associahedron and triangulations of the $n$-gon. Eur. J. Combin., 10 (1989), 551-560.

15. D. Orden, F. Santos, B. Servatius, and H. Servatius. Combinatorial pseudo-triangulations. Preprint 2003. http: //arxiv.org/abs/math. CO/0307370.

16. M. Pocchiola and G. Vegter. Pseudo-triangulations: theory and applications. In Proc. 12th Annual ACM Symposium on Computational Geometry, pages 291-300. ACM Press, New York, May 1996.

17. M. Pocchiola and G. Vegter. Topologically sweeping visibility complexes via pseudo-triangulations. Discrete Comput. Geom., 16 (1996) 419-453.

18. M. Pocchiola and G. Vegter. The visibility complex. Int. J. Comput. Geom. Appl., 6 (1996), 279-308. 
19. G. Rote, F. Santos, and I. Streinu. Expansive motions and the polytope of pointed pseudo-triangulations. In B. Aronov, S. Basu, J. Pach, and M. Sharir, editors, Discrete and Computational Geometry-The Goodman-Pollack Festschrift, pp. 699-736. Algorithms and Combinatorics, volume 25. Springer-Verlag, Berlin, 2003.

20. B. Speckmann and C. D. Tóth. Allocating vertex $\pi$-guards in simple polygons via pseudo-triangulations. In Proc. 14th ACM-SIAM Symposium on Discrete Algorithms (SODA), pp. 109-118, 2003.

21. I. Streinu. A combinatorial approach to planar non-colliding robot arm motion planning. In Proc. 41 st Annual Symposium on Foundations of Computer Science (FOCS 2000), Redondo Beach, California, pages 443-453, 2000.

22. G. M. Ziegler. Lectures on Polytopes. Springer-Verlag, New York, 1995.

Received May 29, 2003, and in revised form May 5, 2004. Online publication November 15, 2004. 